\section{Effects of isolated and enriched rearing on response inhibition*}

\author{
BRUCE R. OUGH, WILLIAM W. BEATTY, and JAMSHID KHALILI \\ North Dakota State University, Fargo, N. Dak. 58102
}

Rats reared socially in an enriched environment exhibited lower response rates and higher efficiency scores on a DRL-20 task than littermates reared in social isolation. A second experiment showed that differential rearing did not alter response rates for food reward on CRF. These findings support the hypothesis that early social isolation impairs the capacity to inhibit irrelevant responses.

Within the last 20 years, many studies have investigated the effects of exposing animals to rearing environments varying the amount of sensory and social stimulation. These experiments have generally shown that early social isolation impairs performance on a variety of learning tasks in several species (e.g., Forgays \& Read, 1962, Fuller, 1967). These learning deficits have usually been attributed to the operation of either or both of two factors: (1) increased emotional reactivity to the novel aspects of the test situation (Denenberg, 1967; Fuller, 1967), or (2) retarded development of perceptual abilities (Forgays \& Read, 1962; Hebb, 1949).

A third possibility that has received little theoretical attention is that early social isolation produces a primary defect in response inhibition. Since learning obviously requires the elimination of responding to nonreinforced stimuli, any defect in the ability to inhibit irrelevant responding could account for the deficits observed in socially isolated animals on a variety of problem-solving tasks. Some data exist which appear to support the failure of response inhibition hypothesis. Rats and beagles reared in social isolation reverse a spontaneous or acquired spatial preference more slowly than animals reared with social experience (Fuller, 1966; Hitt \& Gerall, 1966). Social isolation also greatly reduces spontaneous alternation in rats (Kirkby \& Kirkby, 1968) and impairs passive avoidance of a candle flame (Lore, 1969). On the other hand, Kirkby (1970) failed to observe any effect of social isolation on a step-through passive avoidance task motivated by shock. The present experiment was performed to provide a more direct test of the failure of the response inhibition hypothesis by comparing the performance of rats reared in either an enriched

*We thank Tim Shirk, Bruce Dahl, Ray Cleven, and Jay Gunkelman for technica assistance. environment or an isolated environment on a DRL-20 task.

\section{EXPERIMENT 1}

Subjects albino rats ( 10 for each environment grouping) were obtained from the breeding stock at NDSU's Psychology Department. The two constituent litters were weaned at 21 days of age, sexed, and assigned to enriched environment or socially isolated groups so that each group contained an equal number of rats from each litter.
Twenty infant Holtzman-derived
Apparatus

The enriched environment rearing cage was constructed by lining a 1 $x 2 \times 4 \mathrm{ft}$ wooden frame with $1 / 2$-in. hardware cloth on all external sides. Pans covered with wood shavings were inserted under the elevated cage to absorb droppings. The environment contained such manipulative objects as a transparent plastic maze, tunnels, a seesaw, running wheels, ladders, and abundant food and water. The isolated environment animals were housed singly in 10 standard lab cages. No effort was made to restrict the visual or auditory stimulation available to the isolates, but the rats were not handled or disturbed except as necessary for feeding and cleaning.

Four Gerbrands operant conditioning chambers housed in sound-attenuating enclosures were used for all tests. Relay circuitry programmed conditions and recorded performance.

\section{Procedure}

At 70 days of age, the enriched environment group was placed in individual cages, and all $S$ s were reduced to $80 \%-85 \%$ of their ad lib

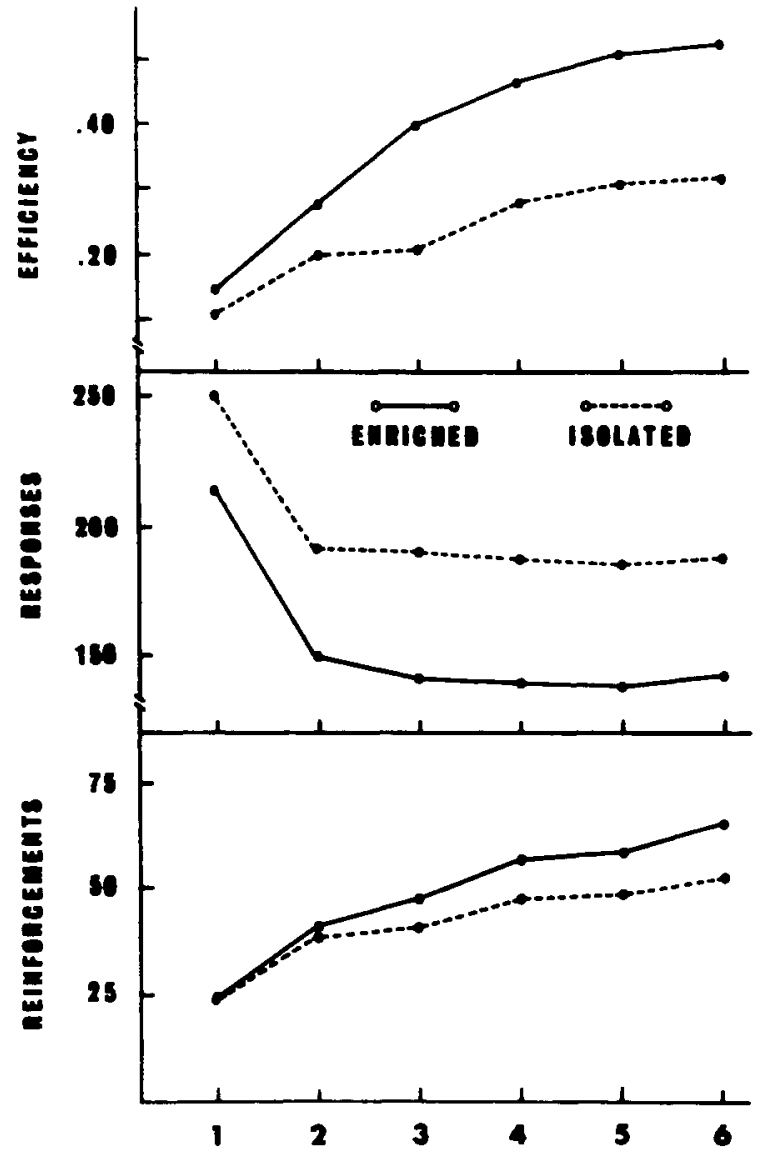

DLOCXS OF fIVE SESSIONS

Fig. 1. Mean efficiency (reinforcements/responses), response rate, and reinforcement rate per session. Each data point is the average of five sessions. 
Table 1

Mean Number of Barpresses Per Session on CRF

\begin{tabular}{lcc}
\hline & Enriched & Isolated \\
\hline Males & 216.5 & 197.3 \\
Females & 180.8 & 156.0 \\
\hline
\end{tabular}

weight for the duration of the experiment. At this age, mean ad lib body weights were, respectively: male enriched $=300.8 \mathrm{~g}$; male isolated $=$ $315.2 \mathrm{~g}$; female enriched $=232.8 \mathrm{~g}$; female isolated $=221.4 \mathrm{~g}$. Only the sex difference was significant.

At 77 days, all Ss were shaped to barpress on a CRF schedule for $45-\mathrm{mg}$ Noyes pellets until they met a criterion of 50 reinforcements in a single session. This required from one to three 40 -min sessions; there were no significant differences between groups in number of sessions required for pretraining. Upon reaching criterion on CRF, the Ss were switched to a DRL-20 schedule on the following day and were tested for 30 daily sessions. Results

As seen in the upper panel of Fig. 1 , rats raised in the enriched environment exhibited more efficient performance on the DRL-20 task than the socially isolated animals $(\mathrm{F}=6.45, \mathrm{df}=1 / 16$, $p<.025)$. The analysis of variance also revealed a significant main effect of sessions ( $F=20.80, \quad d f=29 / 464$, $\mathrm{p}<.001)$ and a significant Environment by Sessions interaction $(F=2.38, \mathrm{df}=29 / 464, \mathrm{p}<.001)$.

The difference in efficiency between the two groups arose primarily because of the increased response rates by the socially isolated group as seen in the middle panel of Fig. 1. Analysis of these data revealed significant effects of rearing environment $(F=7.93, \quad d f=1 / 16$, $\mathrm{p}<.025)$ and sessions $(\mathrm{F}=19.53$, $\mathrm{df}=29 / 464, \quad \mathrm{p}<.001)$. The socially isolated group also earned fewer reinforcements (lower panel of Fig. 1), but this effect was not significant $(F=2.12)$. There was no sex difference on any measure (all Fs $<1$ ).

\section{EXPERIMENT 2}

The findings of the first experiment lent support to the notion that early social isolation impairs the ability of animals to withhold irrelevant responding, but the increased responding and reduced efficiency of the isolates could also be explained if they were more responsive to the positive incentive properties of the reinforcement used. If this were the case, isolates should press more rapidly for food than rats reared in an enriched environment. This experiment examined this possibility by comparing CRF response rates of rats raised in isolated and in enriched environments.

\section{Method}

Infant Holtzman-derived rats were differentially reared from 21 to 70 days of age, as in the first experiment. The number of rats in each condition were: enriched males $(N=4)$; isolated males $(\mathrm{N}=7)$; enriched females $(\mathrm{N}=5)$; isolated females $(\mathrm{N}=4)$. At 70 days of age, the rats in the enriched environment group were transferred to single cages, and all animals were reduced to $80 \%-85 \%$ of their free feeding weights. They were then shaped to barpress for $45-\mathrm{mg}$ Noyes pellets in 1-2 sessions and tested for 14 30-min sessions, during which Noyes pellets were available on CRF. Results

Table 1 shows the average number of responses during the entire $1 / 2$-h session pooled over all 14 sessions. Males earned more reinforcements than females $(F=7.53, \mathrm{df}=1 / 16$, $p<.025$ ), but isolated rats of both sexes earned somewhat fewer rewards than rats reared in the enriched environment $\quad(F=2.46$ $.10<\mathrm{p}<.20)$. Since it seemed possible that satiation effects might have masked differences due to differential rearing, performance during the first $5 \mathrm{~min}$ of the last three CRF sessions was also examined. Again, isolates earned slightly fewer rewards, but the trend was not significant.

\section{DISCUSSION}

The present findings demonstrate that rats reared in social isolation are deficient in the elimination of maladaptive responses as required by the DRL schedule when compared to animals raised in an enriched and socially stimulating environment. The data clearly demonstrate that the decreased efficiency caused by social isolation results from increased response rates. The failure to observe a significant difference in the number of reinforcements may possibly be accounted for by the fact that the reduced response rate required by the DRL schedule also tends to limit opportunities for reinforcement. Thus, the general pattern of results is as predicted by the failure of the response inhibition hypothesis.

It should be noted that the effects of social isolation on DRL behavior cannot be accounted for in terms of changes in motivation produced by the differential rearing treatments. Rats reared in isolated or enriched environments were similar in body weight at the end of rearing and responded at comparable rates for reinforcement on a CRF schedule. These findings can also be accounted for by other explanations of the effects of early social isolation that emphasize increased emotional reactivity to the novel aspects of the learning situation (Fuller, 1967), increased exploratory activity (Woods, Fiske, \& Ruckelshaus, 1961), or the failure of perceptual development (Forgays \& Read, 1962). However, the failure of the response inhibition hypothesis has the advantage of predicting that social isolation will produce deficits in certain learning situations, but not in others. These other explanations do not make such specific predictions.

\section{REFERENCES \\ DENENBERG, $V$. H. Stimulation in infancy, emotional reactivity and exploratory behavior. In D. C. Glass (Ed.), Neurophysiology and emotion. New York: Rockefeller Press, 1967} Pp. 161-189.

FORGAYS. D. G., \& READ, J. M. Crucial periods for free environmental experience in the rat. Joumal of Comparative \& Physiological Psychology, 1962, 55, 816-818.

FULLER, J. L. Transitory effects of experiential deprivation upon reversal learning in dogs. Psychonomic Science, $1966,4,273-274$.

FULLER, J. L. Experiential deprivation and later behavior. Science, 1967, 158, 1645-1652.

HEBB. D. O. The arganization of behavior. New York: Wiley, 1949

HITT, J. C., \& GERALL, H. D. Simple and complex learning in rats reared socially or in isolation. Psychonomic Science, 1966, $4,179-180$.

KIRKBY, R. J. Early environmental experience and avoidance learning in the rat. Psychonomic Science, 1970, 19 , $30-31$.

KIRKBY, R. J., \& KIRKBY, J. E. Early environmental experience and spontaneous alternation. Psychological Reports, 1968, 23, 1278.

LORE, R. Pain avoidance behavior of rats reared in restricted and enriched environments. Developmental Psychology, 1969, 1, 482-484.

WOODS, P. J., FISKE, A. S.. \& RUCKELSHAUS, S. I. The effects of drives conflicting with exploration on the problem-solving behavior of rats reared in free and restricted environments. Journal of Comparative \& Physiological Psychology, 1961, 54, 167-169. 